\title{
Evaluation of the TGS TA system for the detection of anti-Toxoplasma antibodies
}

\author{
Olivia Arpino, Annalisa Cianflone, Maria Teresa Manco, Alessia Paganini, Massimo De Paschale, \\ Carlo Agrappi, Paola Mirri, Pierangelo Clerici
}

Microbiology Unit, Legnano Hospital, ASST Ovest Milanese, Legnano, Italy

\section{Summary}

Background and aims. The aim of the present study was to evaluate the new chemiluminescence TGS TA system of Technogenetics (Milan, Italy) for detecting anti-Toxoplasma IgG and IgM antibodies and IgG avidity. The TGS TA system was compared with our chemiluminescence routinely used system, LIAISON XL, supplied by Diasorin (Saluggia, Italy), for the detection of IgG and IgM antibodies. Only in positive IgM samples (retrospective study) and for the IgG avidity (if existent), TGS TA system was compared to an Enzyme Linked Fluorescent Assay (ELFA) test (VIDAS, BioMérieux, Marcy-l'Étoile, France).

Materials and methods. Three hundred and one sera samples, from women who came to our centre for the routine follow up pregnancy, were examined with the TGS TA system and divided in 3 groups according to $\operatorname{IgG}$ and $\operatorname{IgM}$ screening LIAISON XL tests: 106 were non-immune women (Group 1), 100 were pregnant with past infection (Group 2) and 95 were pregnant with positive or equivocal $\operatorname{IgM}$ ( 82 with positive $\mathrm{IgG}$ and 13 with negative $\mathrm{IgG}$ ) (Group 3).

Correspondence: Olivia Arpino, Microbiology Unit, Legnano Hospital, ASST Ovest Milanese, via Papa Giovanni Paolo II, Legnano (MI), Italy. Tel.: +39.0331.449319

E-mail: olivia.arpino86@gmail.com

Key words: Toxoplasma; Pregnancy; IgM; IgG; Avidity.

Contributions: the authors contributed equally.

Conflict of interest: the authors declare no potential conflict of interest.

Received for publication: 18 January 2017

Revision received: 13 March 2017.

Accepted for publication: 13 March 2017.

(C) Copyright O. Arpino et al., 2017

Licensee PAGEPress, Italy

Microbiologia Medica 2017; 32:6583

doi: $10.4081 / \mathrm{mm} .2017 .6583$

This article is distributed under the terms of the Creative Commons Attribution Noncommercial License (by-nc 4.0) which permits any noncommercial use, distribution, and reproduction in any medium, provided the original author(s) and source are credited.
Results. The overall concordance of the $\mathrm{IgG}$ results between LIAISON XL and TGS TA was $99.3 \%$ : $100 \%$ in Group 1, 98\% in Group 2 and 100\% in Group 3. The overall concordance of the IgM results between LIAISON XL and TGS TA was 93.9\%: 100\% in Group 1, 94\% in Group 2 and $82.8 \%$ in Group 3. In Group 3, the concordance between the results of the IgG avidity with the ELFA and TGS TA tests was $81.7 \%$. Comparing the clinical diagnosis obtained with our protocol and that of the TGS TA system, the overall concordance was $92.7 \%: 100 \%$ in Group 1, $92.0 \%$ in Group 2 and $78.9 \%$ in Group 3.

Conclusions. The overall concordance of $\mathrm{IgG}$ antibodies is excellent for both protocols while for IgM antibodies is very high in the first group and lower in the third group, due to the presence of non-specific IgM subjects in this group. The TGS TA avidity test seems to predict ealier the maturation of the IgG compared to the ELFA test since many samples with low avidity with the ELFA were seen with moderate avidity with TGS TA and all those with borderline avidity with the ELFA were seen with high avidity with TGS TA. This system shows to be a valuable tool with overall good clinical correlation and able to clearly identify nonspecific subjects, those with a non-recent infection.

\section{Introduction}

Toxoplasma gondii is an obligate intracellular parasite, the cat is the definitive host and many warm-blooded animals, including humans, are intermediate hosts. The infection by Toxoplasma gondii is generally benign but can be particularly severe during pregnancy, leading to malformations of the foetus or a series of complications in the newborn $(3,6,7,11)$. This is the reason why the antibody screening campaign in pregnancy was implemented $(1,2,5$, $8,10)$ to indicate possible acute infections with the risk of transmission to the foetus (8). Currently, there are several analytical systems for the detection of antibodies, with different level of automation, such as Enzyme-Linked Immunosorbent Assay (ELISA), Chemiluminescence Immunoassay (CLIA) and Enzyme Linked Fluorescent Assay (ELFA) able, depending on the instrument used, to process a large number of samples in a short time. The com- 
mercial tests available generally have excellent sensitivity and specificity levels, showing slight differences in the results, depending on different formulations and on the use of natural, recombinant or synthetic antigens. The extent of these differences must be known. We compared the data obtained with the Technogenetics TGS TA system in the detection of anti-toxoplasma IgG and IgM antibodies and IgG avidity with the results we obtained with our current standard protocol, which consists of the LIAISON XL system from DiaSorin for IgG and IgM antibodies detection, in order to assess the differences that may arise using different systems. In our protocol, the samples are confirmed using an ELFA test and the IgG avidity is determined (if existent) with an ELFA test in the event of positivity for IgM.

\section{Materials and methods}

Three hundred and one sera samples from women who came to our centre for the routine follow-up in pregnancy were examined and divided into the groups below:

Group 1: 106 samples from non-immune women Group 2: 100 samples from women with past infection Group 3: 95 samples from women with positive or equivocal $\operatorname{IgM}(82$ with positive $\operatorname{IgG}$ and 13 with negative $\operatorname{IgG})$.

Routinely, samples are tested using chemiluminescence LIAISON XL system for $\operatorname{IgG}$ and IgM (LIAISON Toxoplasma IgG, IgM, DiaSorin, Saluggia, Italy). In positive subjects for IgM, samples are tested using an ELFA test (VIDAS Toxo IgM, BioMérieux, Marcy l'Etoile, France). The avidity of the IgG (if present) is determined with an ELFA test (VIDAS TOXO IgG AVIDITY, BioMérieux, France).

All samples are tested with TGS TA system for detecting IgG and IgM and, for Group 3, IgG avidity (TGS TA Toxoplasma IgG, Toxoplasma IgM, Toxoplasma IgG Avidity, Technogenetics, Milan, Italy).
The reference values for the different tests are shown in Tables 1 and 2.

Low avidity is suggestive for a possible infection in the past 4 months preceding the sampling for both systems. High avidity indicates an infection occurred more than 4 months before while for borderline (ELFA)/moderate (TGS TA) avidity, the interpretation is equivocal as a recent infection is not excluded but past infection with partial maturation of the $\mathrm{IgG}$ avidity may also be indicated.

This work does not require an ethics statement. We used residual samples after required routine tests have been completed providing that there was no link to the patient's identity.

Toxoplasma IgG and IgM values are compared using the concordance index in the three groups.

Linear regression correlates LIAISON XL and TGS TA Toxoplasma IgG values.

\section{Results}

The overall concordance in the different groups arising from a comparison of the results obtained with the TGS TA and LIAISON XL systems for the detection of IgG and IgM is shown in Table 3.

The linear regression obtained from the comparison of all the IgG values of the three groups with the LIAISON XL and TGS TA systems is shown in Figure 1.

The discordant samples for IgM (30) in Group 3 were examined with the ELFA IgM test; 19 were negative, 5 positive and 6 equivocal (Table 4).

Further, in the 82 samples with IgG in Group 3, the avidity was determined with both ELFA and TGS TA (Table 5).

As far as the clinical assessment is concerned, the TGS TA correctly considered all the subjects as non-immune in Group 1 (non-immune women). $100 \%$ of clinical concordance.

Table 1. Reference values for the anti-Toxoplasma IgG and IgM tests with the LIAISON XL, ELFA and TGS TA systems.

\begin{tabular}{|c|c|c|c|c|c|}
\hline \multirow[t]{2}{*}{ Reference values } & \multicolumn{2}{|c|}{ IgG } & \multicolumn{3}{|c|}{$\operatorname{Ig} M$} \\
\hline & $\begin{array}{c}\text { LIAISON XL, } \\
\text { IU/mL }\end{array}$ & $\begin{array}{l}\text { TGS TA, } \\
\text { IU/mL }\end{array}$ & $\begin{array}{l}\text { LIAISON XL, } \\
\text { IU/mL }\end{array}$ & $\begin{array}{c}\text { ELFA } \\
\text { (index) }\end{array}$ & $\begin{array}{l}\text { TGS TA, } \\
\text { IU/mL }\end{array}$ \\
\hline Negative & $<7.2$ & $<1.5$ & $<6$ & $<0.70$ & $<15$ \\
\hline Equivocal & $7.2-8.8$ & - & $6-8$ & $0.70-0.90$ & - \\
\hline Positive & $>8.8$ & $\geq 1.5$ & $>8$ & $>0.90$ & $\geq 15$ \\
\hline
\end{tabular}

The LIAISON XL test for IgG is standardised on the Second WHO International Standard of 1980 while the TGS TA test is calibrated against the First WHO International Standard of 2003.

Table 2. Reference values for the anti-Toxoplasma IgG avidity tests with the LIAISON XL and TGS TA systems.

\begin{tabular}{|c|c|c|}
\hline Reference value & & \\
\hline & ELFA (index) & TGS TA (index) \\
\hline Low & $<0.200$ & $<0.100$ \\
\hline Borderline/moderate & $0.200-0.300$ & $0.100-0.150$ \\
\hline High & $>0.300$ & $\geq 0.150$ \\
\hline
\end{tabular}


In Group 2 (pregnant women with past infection), the TGS TA system showed two cases, which had weakly positive $\operatorname{IgG}$ values with LIAISON XL (9.3 and $13.2 \mathrm{IU} / \mathrm{mL}$ ) as non-immune.

However, in the same group, the TGS TA test showed 6 subjects with positive IgM (negative to the LIAISON XL) and 5 of them were confirmed with the ELFA IgM test.

These patients had high avidity with both tests except for one which was insufficient to carry out the TGS TA avidity test. $92 \%$ of clinical concordance.
The samples with our protocol were typified as follows in Group 3 (IgM samples positive or equivocal to the LIAISON XL IgM): 17 patients with recent infection (ELFA IgM positive and low ELFA avidity) of whom 4 with negative IgG; 4 with a non-determinable infection (ELFA IgM positive and intermediate avidity to ELFA); 42 patients with past infection (ELFA IgM positive and high ELFA avidity); 23 patients with IgM considered non-specific (IgM not confirmed with the ELFA test and with high ELFA avidity); 9 non-immune patients (IgM supposed non-specific: ELFA IgM negative without $\operatorname{IgG}$ ).

TOXOPLASMA IgG: TGS TA vs LIAISON XL

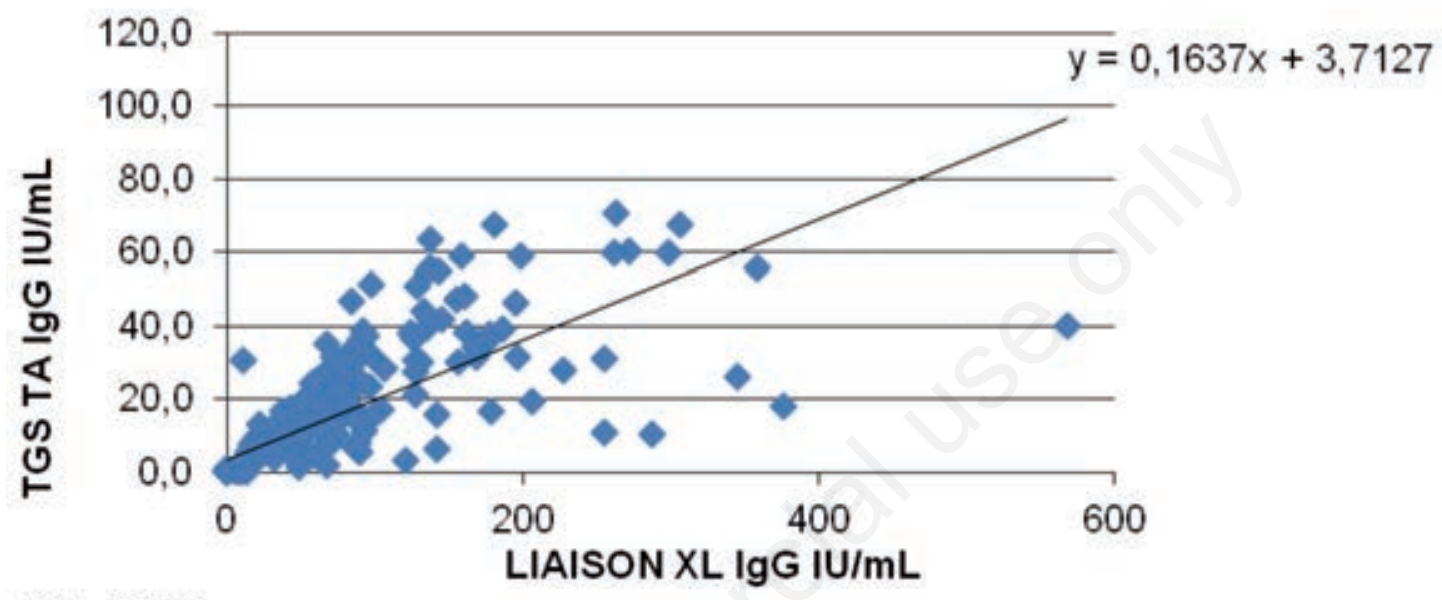

$\mathrm{R} 2=0.596$

Figure 1. Linear regression between TGS TA and LIAISON XL of the anti-Toxoplasma IgG (IU/mL).

Table 3. Overall concordance of LIAISON XL and TGS TA for the detection of anti-Toxoplasma IgG and IgM in samples from pregnant women.

$\begin{array}{lllll}\text { TGS TA neg } & \lg G \quad \text { TgM TA pos } & \text { TGS TA neg } & \text { TGS TA pos }\end{array}$

\section{Overall concordance (301 samples from pregnant women)*}

\begin{tabular}{lcccc} 
LIAISON XL neg & 119 & 0 & 200 & 6 \\
LIAISON XL pos & 2 & 180 & 10 & 48 \\
\hline LIAISON XL eq & - & - & 20 & 17 \\
Group 1 concordance (106 samples from pregnant non-immune women) ${ }^{* *}$ & 0 & 106 & 0 \\
LIAISON XL neg & 106 & 0 & 0 & 0 \\
LIAISON XL pos & 0 & &
\end{tabular}

\begin{tabular}{lcccc} 
LIAISON XL neg & 0 & 0 & 94 & 6 \\
LIAISON XL pos & 2 & 98 & 0 & 0 \\
\hline Group 3 concordance $(95$ samples from pregnant women with positive or equivocal IgM to the LIAISON) ****
\end{tabular}

\begin{tabular}{lcccc} 
LIAISON XL neg & 13 & 0 & 0 & 0 \\
LIAISON XL pos & 0 & 82 & 10 & 48 \\
\hline LIAISON XL eq & - & - & 20 & 17 \\
\hline$*$ O & & 20
\end{tabular}

${ }^{*}$ Overall concordance (excluding equivocal cases): IgG 99.3\%; IgM 93.9\%. ${ }^{* *}$ Overall concordance: IgG 100\%; IgM 100\%. *** Overall concordance: IgG 98\%; IgM 94\%. **** Overall concordance (excluding equivocal cases): $\lg \mathrm{G}$ 100\%; IgM 82.8\%. 
The typing obtained with the TGS TA system (IgG, IgM and $\mathrm{IgG}$ avidity) in these sub-groups is shown in Table 6.

Considering all three groups, the clinical concordance is shown in Table 7.

\section{Discussion and Conclusions}

In conclusion, the concordance between LIAISON XL and TGS TA system for IgG is excellent in all groups (99.3\%). The results of the IgG with TGS TA correlate well as a qualitative result with the LIAISON XL test but, considering the quantitative values in IU/mL, the linear correlation is low with an R2 of 0.596 . This evidence may be due to the fact that the two tests are calibrated towards different international standards.

The concordance of the tests for IgM is excellent in the first group. In the third group, the discordance is greater, also because in this group there were subjects with non-specific IgM.

The discrepancies may also be influenced by different antigen preparation in the two systems, but the majority of the samples scored as non-specific with our current protocol,

Table 4. Results of the ELFA test on 30 samples equivocal or positive to an IgM LIAISON XL test and negative with the TGS TA IgM.

\begin{tabular}{lccc}
\hline IgM positive or equivocal to the LIAISON XL system & ELFA & TIAISON XL TA \\
Negative & 0 & 19 & 30 \\
Positive & 10 & 5 & 0 \\
\hline Equivocal & 20 & 6 & 0 \\
\hline
\end{tabular}

Table 5. The concordance between the ELFA and TGS TA tests for IgG avidity in 82 subjects positive or equivocal (with positive IgG) to the LIAISON XL IgM.

\begin{tabular}{lcccc} 
& \multicolumn{2}{c}{ TIGS TA avidity } & High & Total \\
ELFA avidity & Low & & & \\
Low & & 10 & 1 & 13 \\
Borderline & 2 & 0 & 4 & 4 \\
High & 0 & 0 & 65 & 65 \\
Total & 0 & 10 & 70 & 82 \\
\hline
\end{tabular}

Concordance 81.7\%.

Table 6. Comparison of the clinical interpretations given with the LIAISON/ELFA protocol and TGS TA system.

\begin{tabular}{|c|c|c|c|c|c|}
\hline LIAISON/ELFA protocol & $\begin{array}{c}\text { Probable recent } \\
\text { infection }\end{array}$ & $\begin{array}{l}\text { Indeterminate } \\
\text { infection }\end{array}$ & $\begin{array}{c}\text { TGS TA system } \\
\text { Probable non-recent } \\
\text { infection }\end{array}$ & Non immune & Total \\
\hline Probable recent infection & 5 & 10 & 1 & 1 & 17 \\
\hline Indeterminate infection & 0 & 0 & 4 & 0 & 4 \\
\hline Probable non-recent infection & 0 & 0 & 65 & 0 & 65 \\
\hline Non immune & 4 & 0 & 0 & 5 & 9 \\
\hline Total & 9 & 10 & 70 & 6 & 95 \\
\hline
\end{tabular}

Concordance $78.9 \%$.

Table 7. Clinical concordance between the LIAISON/ELFA protocol and the TGS TA system considering all three groups.

\begin{tabular}{|c|c|c|c|c|c|}
\hline LIAISON/ELFA protocol & $\begin{array}{l}\text { Probable recent } \\
\text { infection }\end{array}$ & $\begin{array}{l}\text { Indeterminate } \\
\text { infection }\end{array}$ & $\begin{array}{c}\text { TGS TA system } \\
\text { Probable non-recent } \\
\text { infection }\end{array}$ & Non immune & Total \\
\hline Probable recent infection & 5 & 10 & 1 & 1 & 17 \\
\hline Indeterminate infection & 0 & 0 & 4 & 0 & 4 \\
\hline Probable non-recent infection* & 0 & 0 & 162 & 2 & 164 \\
\hline Non immune & 4 & 0 & 0 & 111 & 115 \\
\hline TOTAL & 9 & 10 & 167 & 114 & 300 \\
\hline
\end{tabular}

Total concordance 92.7\%. *One sample insufficient quantity for TGS TA avidity. 
were indicated as negative in the TGS TA system, suggesting good specificity. Furthermore, the TGS TA system does not have a grey area around the cut-off unlike the LIAISON XL and ELFA tests, therefore the calculation of the concordance suffers from this limitation. Another limitation of the study is the lack of cases of recent Toxoplasma infection but this is due to low incidence of acute infection in pregnant women in our area (4) that makes observation of recent infection rare.

Most of the samples with low avidity with ELFA test were seen as moderate avidity to the TGS TA test while all samples borderline to the ELFA test were seen with high avidity to the TGS TA test, suggesting that TGS TA system may predict the maturation of $\operatorname{IgG}$ in advance with respect to the ELFA test. The samples analysed came from asymptomatic pregnant women so the clinic was unable to provide additional indications on the status of the infection. Further investigations would be necessary for both avidity tests with an indeterminate result.

Finally, the overall clinical concordance is good; the TGS TA system clearly indicates subjects with probable non-specific IgM or non-recent infection but, while it indicates additional possible recent infections (four) on one hand, on the other, it indicates some less (two) than our protocol.

In conclusion, TGS TA is a useful system for the evaluation of Toxoplasma infection.

\section{References}

1. Agence Nazionale d'Accréditation et d'Evaluation en Santé. La surveillance biologique de la femme enceinte en bonne santé et sans antécédents pathologiques. Paris: ANAES; 1996.
2. Aspöck H, Pollak A. Prevention of prenatal toxoplasmosis by serological screening of pregnant women in Austria. Scand J Infect Dis 1992;84:32-7.

3.Daffos F, Forestier F, Capella-Pavlovsky M, et al. Prenatal management of 746 pregnancies at risk for congenital toxoplasmosis N Engl J Med 1988;318:271-5.

4. De Paschale M, Agrappi C, Clerici P, et al. Seroprevalence and incidence of Toxoplasma gondii infection in the Legnano area of Italy. Clin Microbiol Infect 2008;14:186-9.

5.De Paschale M, Agrappi C, Manco MT, et al. Implementation of screening for Toxoplasma gondii infection in pregnancy. J Clin Med Res 2010;2:112-6.

6. Desmonts G, Couvreur J. Congenital toxoplasmosis. Prospective study of the outcome of pregnancy in 542 women with toxoplasmosis acquired during pregnancy. Ann Pediatr 1984;31:805-9.

7. Dunn D, Wallon M, Peyron F, et al. Mother to child transmission of toxoplasmosis: risk estimates for clinical counselling. Lancet 1999;353:1829-33.

8. Hengst P. Screening for toxoplasmosis in pregnant women: presentation of a screening programme in the former East Germany and the present status in Germany. Scand J Infect Dis 1992;84:38-42.

9. Meroni V, Genco F. Toxoplasmosis in pregnancy: evaluation of diagnostic methods. Parassitologia 2008;50:51-3.

10. Remington JS, McLeod R, Desmonts G. Toxoplasmosis. In: Remington JS, Klein JO, eds. Infectious diseases of the fetus and the newborn infant. 4th Ed. Philadelphia: WB Saunders; 1995. pp 140-243.

11. Tenter AM, Heckerroth AR, Weiss LM. Toxoplasma gondii: from animals to human. Int J Parasitol 2000;30; 1217-58. 